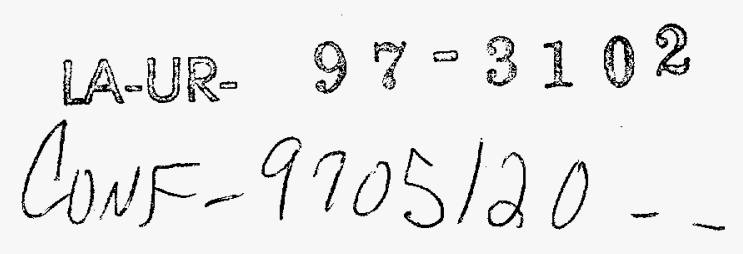

\title{
Variation of High-Power Aluminum-Wire Array Z-Pinch Dynamics with Wire Number, Load Mass, and Array Radius
}

\author{
T. W. L. Sanford, R. C. Mock, B. M. Marder, T. J. Nash, \\ and R. B. Spielman \\ Sandia National Laboratories, Albuquerque, NM 87185 \\ DEC 011937 \\ D. L. Peterson and N. F. Roderick \\ Los Alamos National Laboratory, Los Alamos, NM 87545 \\ OST! \\ J. H. Hammer and J. S. De Groot \\ Lawrence Livermore National Laboratory, Livermore, CA 94550 \\ D. Mosher \\ Naval Research Laboratory, Pulsed Power Physics Branch, Washington, DC 20375
}

K. G. Whitney and J. P. Apruzese

Naval Research Laboratory, Radiation Hydrodynamics Branch, Washington, DC 20375

\begin{abstract}
A systematic study of annular aluminum-wire z-pinches on the Saturn accelerator shows that the quality of the implosion, (as measured by the radial convergence, the radiated energy, pulse width, and power), increases with wire number. Radiation magnetohydrodynamic (RMHC) $x y$ simulations suggest that the implosion transitions from that of individual wire plasmas to that of a continuous plasma shell when the interwire spacing is reduced below $\sim 1.4 \mathrm{~mm}$. In this "plasma-shell regime," many of the global radiation and plasma characteristics are in agreement with those simulated by 2D-RMHC $\mathrm{rz}$ simulations. In this regime, measured changes in the radiation pulse width with variations in load mass and array radius are consistent with the simulations and are explained by the development of 2D fluid motion in the rz plane. Associated variations in the $\mathrm{K}$-shell yield are qualitatively explained by simple radiation-scaling models.
\end{abstract}

\section{INTRODUCTION}

Increasing the symmetry of cylindrical aluminum-wire arrays by significantly increasing wire number (and decreasing the associated interwire gap spacing and wire size) has resulted in the highest $\mathrm{x}$-ray power $(\sim 40 \mathrm{TW}$, Fig. 1$)$ and narrowest $\mathrm{x}$-ray pulse-width ( $5 \mathrm{~ns}$, Fig. 2) measured for aluminum-wire implosions (1) on the 20-TW Saturn accelerator (2). For gap spacings less than $1.4 \mathrm{~mm}$ (Fig. 1), a dramatic increase is seen in the peak power when plotted against the interwire gap. The similar results seen in Fig. 1 for different initial implosion radii (R) may 


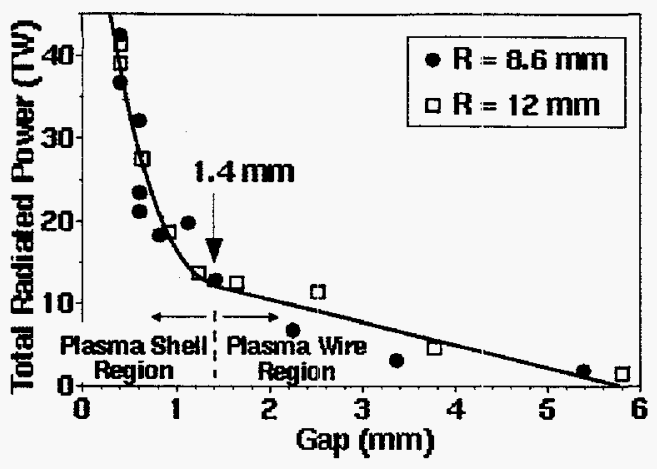

FIGURE 1. Total radiated power measured by bolometer (corrected for pulse shape distortion, spectral response, and viewing angle) versus interwire gap for two initial array radii $R$.

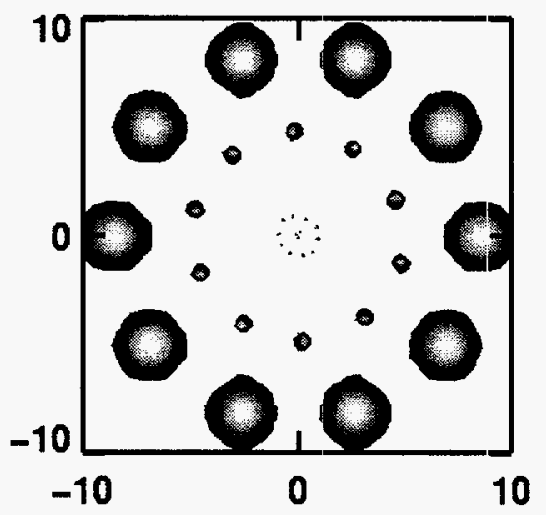

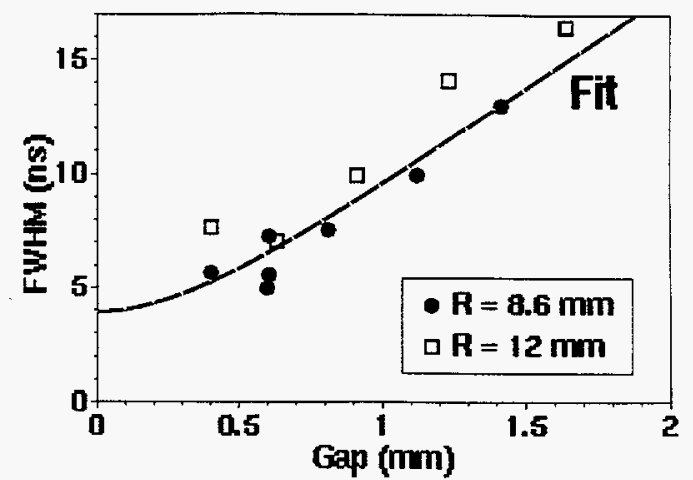

FIGURE 2. Estimated total-radiated-power pulse width (measured in XRD filtered by $1-\mu \mathrm{m}$ Kimfol) versus interwire gap (g) corresponding to data of Fig. 1. Fit $=\sqrt{(3.9 \pm 2)^{2}+[(8.8 \pm 2) \mathrm{g}]^{2}}$.

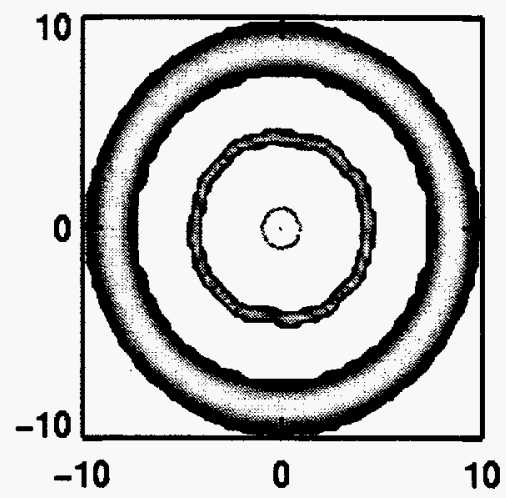

FIGURE 3. $x y-R M H C$ simulations for $(A)$ a wire-plasma regime $(N=10$ wires $)$ and $(B)$ a plasma-shell regime ( $\mathrm{N}=40$ wires) $8.6-\mathrm{mm}$ implosion at $86,11,3$, and 0 ns before stagnation. Azimuthal orientations at different times are not correlated.

be explained by the fact that the larger radius implosions have both increased total energy and pulsewidth (Fig. 2), resulting in similar powers to those at smaller radius. For loads with large number of wires and small interwire gaps $(<1.4 \mathrm{~mm})$, the dramatic improvement in pinch quality may be associated with the effect seen in xy ("end-on") RMHC calculations (1) of the merger of the separate wire plasmas to form a single plasma shell (Fig. 3). Here such loads are designated as being in the plasma-shell regime, and those with larger interwire spacings as being in the plasma-wire regime. In general, our measurements and analyses show that as the wire number increases, the quality of the implosion and the radiated power increase monotonically. Similar effects are found for tungsten wire implosions as discussed in Refs. 3 and 4 and papers at this conference. 


\section{DISCLAIMER}

This report was prepared as an account of work sponsored by an agency of the United States Government. Neither the United States Government nor any agency thereof, nor any of their employees, makes any warranty, express or implied, or assumes any legal liability or responsibility for the accuracy, completeness, or usefulness of any information, apparatus, product, or process disclosed, or represents that its use would not infringe privately owned rights. Reference herein to any specific commercial product, process, or service by trade name, trademark, manufacturer, or otherwise does not necessarily constitute or imply its endorsement, recommendation, or favoring by the United States Government or any agency thereof. The views and opinions of authors expressed herein do not necessarily state or reflect those of the United States Government or any agency thereof. 


\section{DISCLAMMER}

Portions of this docament may be illegible in electronic image products. Images are produced from the bert available original docoment 
Prior to these aluminum experiments, wire-array loads had been optimized for $\mathrm{x}$-ray energy and power output only in the small-wire-number $(\mathrm{N} \leq 40)$ wireplasma regime (5-7), where the calculations now indicate (1) the array may implode inward as a set of discrete wire plasmas. The substantial improvement in implosion quality and associated radiated power that occurs in the plasma-shell regime suggested that a reexamination of the radius and mass conditions that maximize the radiation output in this regime would be instructive. Measurements of the more-nearly-2D, azimuthally-symmetric plasma-shell-implosion could then be more realistically compared with $\mathrm{rz}$ ("side on") RMHC simulations to gain insight into fundamental physical processes, free of the large azimuthal in homogeneities that have limited previous detailed comparisons. Moreover, IDRMHC simulations that utilize detailed radiation models not employed in the 2D codes, and often used to infer the measured plasma conditions from the measured $\mathrm{X}$-ray spectral data, become more realistic.

Additionally, a need existed to assess the effect that longer implosion times would have on the pinch quality expected for the recently commissioned, 45-TW PBFA-Z accelerator $(8,9)$. For PBFA-Z, the optimal coupling of accelerator energy to the load was designed to occur for implosion times on the order of 100 ns, roughly twice that found for loads on the Saturn accelerator. Therefore, after the initial wire-number experimental series (1), two additional experimental series were conducted both to re-examine the conditions that optimize the XUV and Kshell $\mathrm{x}$-ray output and to explore the longer-implosion-time regime using largewire-number, plasma-shell-regime loads. In one series, the radius was held fixed, and the mass was varied (10). In the other series, the mass was held fixed near the mass that optimized the $\mathrm{x}$-ray output, and the radius was varied (11). These two new experimental series enabled the $\mathrm{x}$-ray production to be measured in conjunction with the implosion quality over implosion durations of 40 to $90 \mathrm{~ns}$.

A schematic of the generic arrangement used for all of the experiments and the equivalent-circuit model that was used as driver to the RMHC simulations are shown in Fig. 4. The particular arrangements and circuit model are discussed in detail elsewhere $(1,12$, and 13$)$. The experiments were simulated with the multiphoton-group 2D-Lagrangian-code (L-RMHC) (14), which modeled $1 \mathrm{~mm}$ of the pinch length (12); the three-temperature 2D-Eulerian-code (E-RMHC) $(15,16)$, which modeled the entire pinch length; and the detailed-multi-spectral 1DLagrangian-code (1D-RMHC) (17). In the initial wire-number scan, the array mass and radius were fixed at either $\sim 0.62 \mathrm{mg}$ and $8.56 \mathrm{~mm}$ or $0.84 \mathrm{mg}$ and 12 $\mathrm{mm}$ for the 2-cm-long loads. The wire number was changed incrementally from 10 to 192 by varying the diameter of the individual wires from 37 to $10 \mu \mathrm{m}$, permitting interwire spacings from 6 to $0.4 \mathrm{~mm}$ to be explored. For the mass scan, the radius was fixed at $12 \mathrm{~mm}$ and the mass was varied between 0.42 and $3.36 \mathrm{mg}$ by changing the diameter and the number of wires. For masses of 0.84 $\mathrm{mg}$ and greater, the number of wires was held at 192 (0.4 mm interwire spacing), 
and the wire diameter was varied between 10 and $20 \mu \mathrm{m}$ in $2.54-\mu \mathrm{m}$ steps. Because

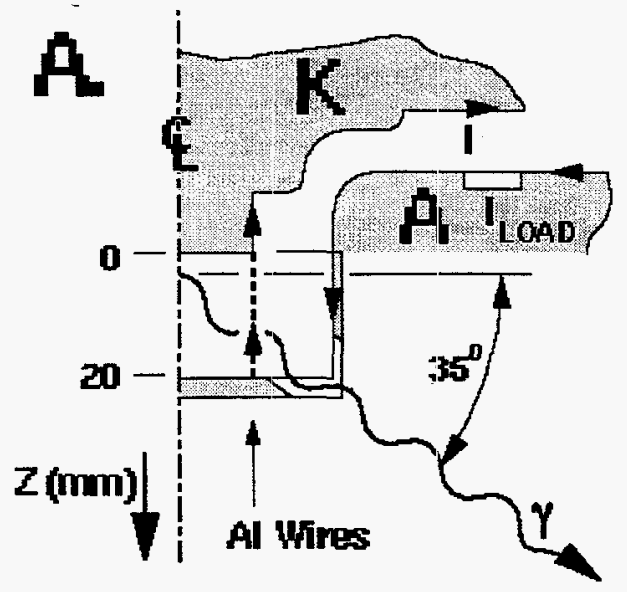

B. ZORKModet

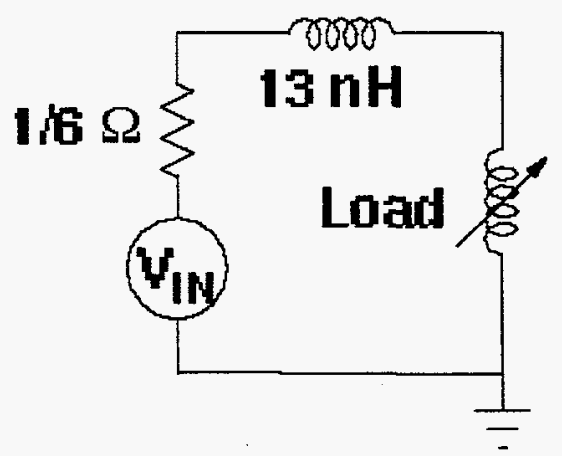

FIGURE 4. (A) Schematic of experimental arrangement showing (B) equivalent circuit. The 13-nH corresponds to the system inductance up to the initial wire location.

smallest diameter aluminum wire available was $10 \mu \mathrm{m}$, the mass region below $0.84 \mathrm{mg}$ was necessarily explored by reducing the number of wires, resulting in an increase in the interwire spacing to $0.8 \mathrm{~mm}$. For the radius scan, the array mass was held fixed at $0.6 \mathrm{mg}(136,10.2-\mu \mathrm{m}$-diameter wires), where high powers had been produced in the mass scan. These arrays had interwire spacings that varied from $0.4 \mathrm{~mm}$ for the smallest, to $0.94 \mathrm{~mm}$ for the largest array diameter.

In this paper, the results of the initial wire-number scan are briefly reviewed. The plasma and radiation generated in the plasma-shell regime are then characterized and compared with the L-RMHC, E-RMHC, and 1D-RMHC simulations. Lastly, the variation of these plasma and radiation characteristics with mass and radius in the plasma-shell regime is discussed and contrasted with results produced by the E-RMHC and two K-shell models.

\section{WIRE NUMBER VARIATION}

The improvement in pinch quality with wire number in both geometries $(\mathrm{R}=$ 8.56 and $12 \mathrm{~mm}$ ) is evident in three ways: (A) the $\mathrm{x}$-ray pinhole camera images that show increased radial convergence (Fig. 5A) and associated total increased radiated energy (Fig. 5B), (B) the load-current traces that show an increased inductive current notch at peak compression, and (C) the $\mathrm{x}$-ray pulses that show reduced radiation rise time and pulse width (Fig. 2), and the associated increase in radiated power (Fig. 1). The increase in measured radiated energy is consistent, within a scale factor $(1.3 \pm 0.2)$, with that expected from the increased kineticenergy input predicted by the ZORK coupled circuit and slug-model code (18) (Fig. 5B), using the measured radial convergence from $\mathrm{x}$-ray pinhole photographs (Fig. 5A). For high-wire-number loads, the current and main radiation pulse 
shapes are consistent with those simulated by the $\mathrm{rz} \operatorname{RMHCs}(1,10,11,12,14)$, as is discussed next.

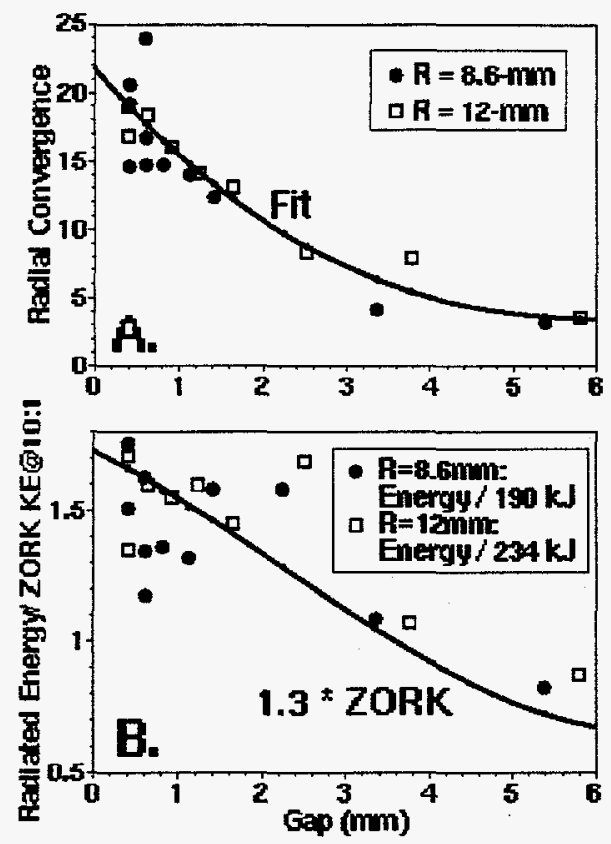

FIGURE 5. (A) Radial convergence determined from time-integrated pinhole images filtered to view $x$ rays greater than $1 \mathrm{keV}$ versus interwire gap $(\mathrm{g})$ : Fit $=22-7.2 \mathrm{~g}+0.9 \mathrm{~g}^{2}-0.0034 \mathrm{~g}^{3}$. Comparison of $(B)$ total radiated energy measured in bolometer (uncorrected for spectral response and viewing angle) normalized by calculated ZORK kinetic energy for a 10-to-1 radial convergence (190 and $234 \mathrm{~kJ}$ for $R=8.6$ and $R=12$ $\mathrm{mm}$, respectively) with 1.3 times the kinetic energy predicted by ZORK using measured radial convergence of Fig. $2 \mathrm{~A}$.

\section{PLASMA-SHELL REGIME CHARACTERISTICS}

Loads in the plasma-shell regime having interwire spacings of less than $\sim 0.6 \mathrm{~mm}$ exhibit both a strong first and weaker second radiation pulse (Fig. 6A) that correlates in time with strong and weaker measured radial convergences (Fig. $6 \mathrm{~B})$. The radial convergence is obtained from radial lineouts of time-resolved $\mathrm{x}$ ray images such as those shown in Fig. 7 for Shot $2235(\mathrm{~m}=1.3 \mathrm{mg}, \mathrm{R}=12 \mathrm{~mm}$, $N=192$ wires) taken during the mass scan, or in Fig. 8 for Shot $2085(\mathrm{~m}=0.6$ $\mathrm{mg}, \mathrm{R}=8.6 \mathrm{~mm}, \mathrm{~N}=90$ wires), taken during the wire-number scan.

In general, by adjusting a random density perturbation $(14,15)$ in a given initial plasma-shell thickness, one can produce rz-RMHC simulations that replicate many of the features measured at the first implosion. This perturbed density seeds the growth of a magnetic Rayleigh-Taylor (RT) instability in the rz plane, which limits the radial convergence. An emission opacity approximation in the simulations, however, allows greater radiation cooling to occur than is physical (12). The cooler plasma has reduced radial expansion following stagnation and the simulated plasma rapidly reaches a quasi-equilibrium without going through the second compression seen experimentally. A reduction in the plasma emissivity, as is done in some of the E-RMHC simulations (to account for the reduced emission from aluminum), nevertheless, permits more realistic peak powers to be simulated and simultaneously generates a second convergence as shown in Figs. 6A and 6B. 


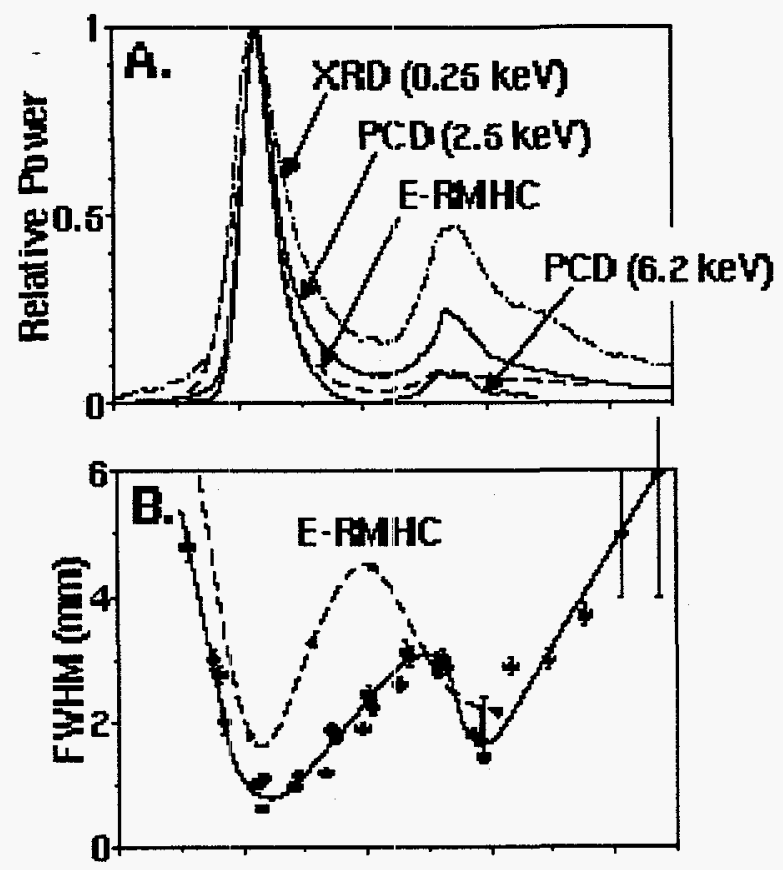

FIGURE 6.

(A) Comparison of radiation pulses measured in XRDs and PCDs sensitive to $x$ rays in bands about $0.25,2.5$, and $6.2 \mathrm{keV}$ with that simulated by the E-RMHC using reduced plasma emissivity for the 90-wire 8.6-mm radius Shot 2094.

(B) Comparison of the pinch diameter measured from fast-framing $x$-ray pinhole cameras (sensitive to $x$ rays greater than $\sim 1 \mathrm{keV}$ ) with the E-RMHC FWHM of the z-averaged radial mass profile associated with the Fig. 6A simulation, for three 90-wire, 8.6- $\mathrm{mm}$ radius Shots 2085, 2094, and 2095.

(C) Comparison of the core electron temperature derived from free-bound slope for Shot 2094 with the average halo electron temperature determined from the ratio of $\mathrm{He}$-like to $\mathrm{H}$-like transitions for Shots 2085, 2094, and 2095.

(D) Opacity corrected, Doppler broadened, Lyman-beta, relative linewidth averaged over Shots 2085, 2094, and 2095. Right-hand scales indicate inferred generalized ion temperatures and associated velocities.

Near peak emission, the radial lineouts of $x$-ray images like that shown in Fig. $8 \mathrm{~A}(0 \mathrm{~ns})$ are well fit by the sum of an intense Gaussian-like core surrounded by a 
diffuse Gaussian-like halo, when integrated over the axial length of the pinch. Radially-resolved images of the $\mathrm{x}$-ray spectrum show that $\mathrm{K}$-shell free-bound

FIGURE 7. Comparison of

A. $-9 \mathrm{~ns}$

B. -7ns
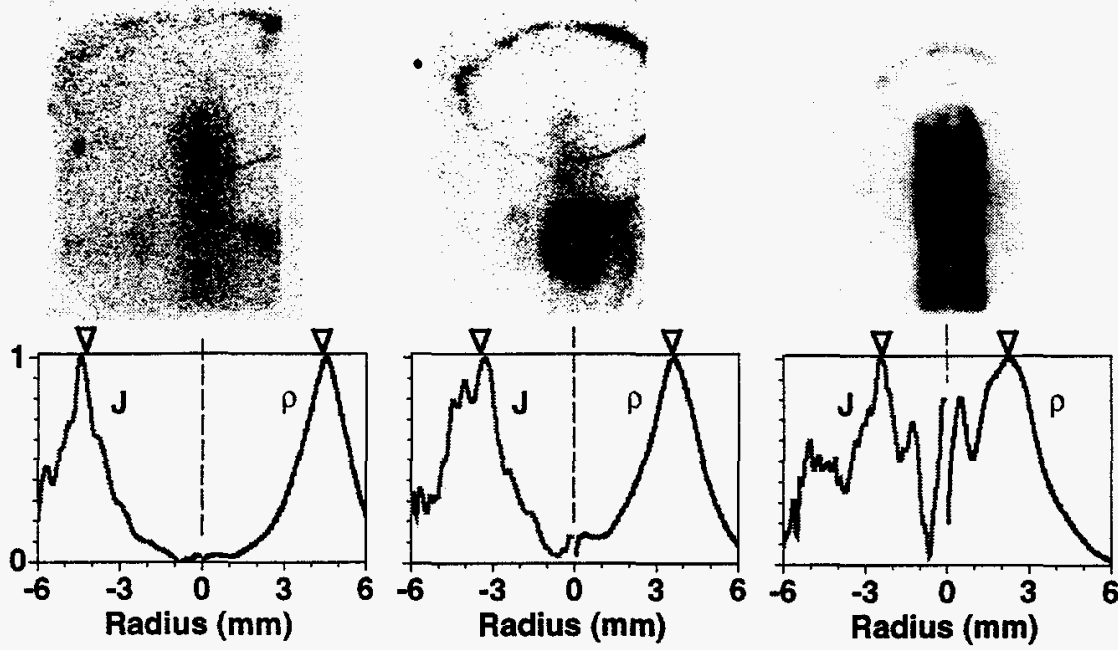

fast-framing $x$ -

ray camera

images [top] (sensitive to 0.2 to $0.3 \mathrm{keV}$ and greater than $1 \mathrm{keV} x$ rays) with E-RMHC simulations [bottom] of mass and current density, $-9,-7$, and -4 ns before peak power for Shot 2235. Arrows correspond to RMS averages.

A. Radial Lineout
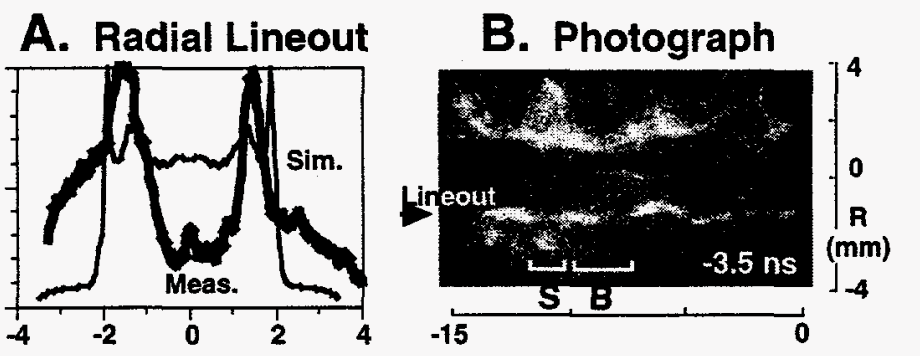

C. Axial Lineout
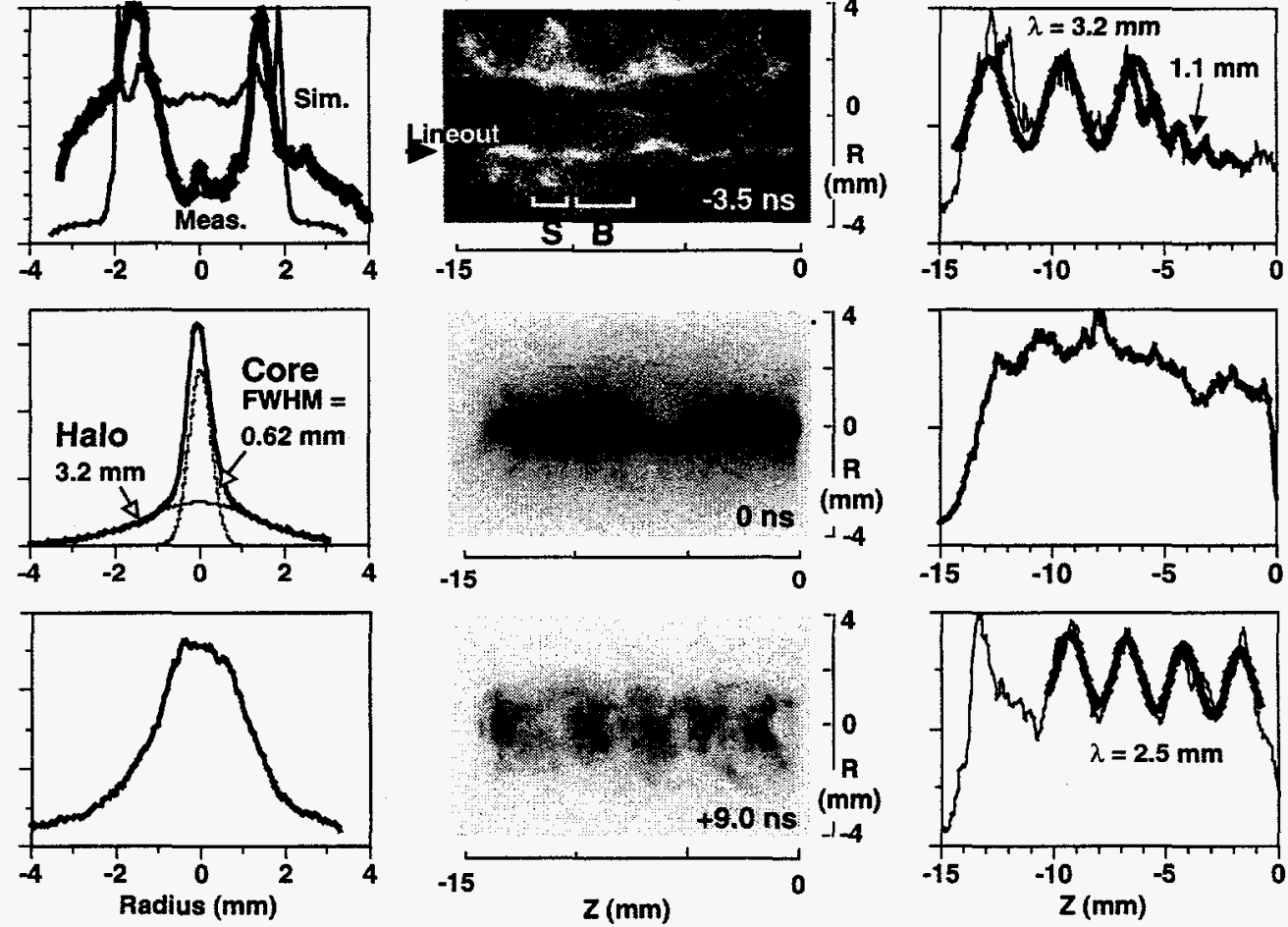
FIGURE 8. Comparison of fast-framing $x$-ray camera images (center) and their associated lineouts -3.5 (including the L-RMHC simulated radial lineout), 0 , and 9 ns with respect to the time of peak power for Shot 2085. Images are sensitive to $x$-rays greater than $1 \mathrm{keV}$. The -3.5 -ns image was also sensitive to 0.2 to $0.3 \mathrm{keV} \times$ rays.

continuum emission originates from the core, whereas $\mathrm{K}$-shell line emission originates from both the core and halo (13), in agreement with 1D-RMHC simulations (19). The time-resolved slope of the optically-thin free-bound emission measured with filtered photoconducting detectors (PCDs) gives a spatially average temperature of the electrons in the core (13), and the ratio of the time-resolved, optically-thick hydrogen-like to helium-like line emissions gives a measure of the electron temperature in the halo (19) (Fig. 6C). The Dopplerbroadened line emission provides an estimate of a generalized ion temperature corresponding to a combination of line-emission shifts caused by the coherent radial motion of the plasma, motion from fluid turbulence, micro instabilities, and intrinsic ion thermal motions (Fig. 6D) (12). The measured hot followed by cooler peaks in the core electron and ion temperatures/velocities correlate in time with the two radiation bursts and radial compressions, as expected for multiple implosions (Fig. 6).

At peak convergence, the temperature of the halo $(\sim 0.4 \mathrm{keV})$ is estimated to be three to four times cooler than that of the core ( $1.4 \mathrm{keV}$ ) (Fig. $6 \mathrm{C})$, indicating the presence of strong temperature gradients. While both line and continuum emissions are generated in the hot plasma core at the time of peak convergence, only the optically-thin continuum $x$ rays escape the plasma directly. The optically-thick line emission is absorbed and reemitted from the cooler regions until the pinch expands and the plasma opacity declines. The high temperatures inferred from the line analysis at times when the plasma is expanding and the core electrons are cooling (Fig. 6C) suggests a lag in recombination (19). That is, the ionization state is likely no longer in equilibrium with the cooled electrons, but reflects instead the earlier state of the plasma when the electrons were hot and the plasma was in equilibrium. The core electrons cool too rapidly in this case for recombination processes to maintain ionization equilibrium. Alternatively, the $\mathrm{rz}$ L-RMHC simulations show rapid stirring of the plasma after stagnation, with development of a boundary between a radiatively dominated, high-density, cooling core and a low-radiating, low-density, hotter halo. The boundary between the hotter and cooler regions has a reversed pressure gradient $(\partial \mathrm{p} / \partial \mathrm{r}>0)$ and is stable, preventing convective mixing across the boundary. Convection does not cross the boundary, allowing a temperature gradient to exist as suggested by the temperature difference measured between the cooler core and hotter halo midway between the two implosions (Fig. 6C).

The early time characteristics of the plasma-shell implosions are illustrated in Fig. 7 and Fig. 8A (-3.5 ns). The rings evident in Fig. 7 are hypothesized to be from a radiating plasma procluced by the electron-current flow into the surface of the imploding plasma shell (Fig. 4A). In support of this hypothesis, the radii of the rings correlate with both the mass-averaged and current-density averaged radii 
calculated by the E-RMHC simulation (Fig. 7). In the simulation, the calculated radially-converging plasma precursor from instability bubble regions (15) begins to stagnate on axis $9 \mathrm{~ns}$ prior to peak emission (Fig. 7A), reaching electron temperatures of $\sim 1 \mathrm{keV}$. As indicated by the simulation and measured, this precursor becomes detectable just at this time. As the stagnation evolves in the simulation, plasma accretes on axis, and together with the inward moving shell the appearance of a hot inward-moving luminescent shell is generated (Fig. 7C). The L-RMHC simulation of such a shell, which takes account of camera sensitivity and $35^{\circ}$ viewing angle (Fig. $\left.4 \mathrm{~A}\right)$, is shown in Fig. 8A ( $-3.5 \mathrm{~ns}$, Sim.) for Shot 2085 (12).

These observations are consistent with the 1D-RMHC analyses (19) that required a cool shell of plasma to implode onto a small amount of precursor plasma to explain the total to $\mathrm{K}$-shell power ratio. In this model, the cool outer shell of plasma is needed to explain the greater than 4-to-1 power ratio measured, and it is also consistent with the spectral analysis $(13,19)$ which found that only $20 \%$ of the mass contributes to the $\mathrm{K}$-shell radiation at the time of peak power. Moreover, the experimentally observed size of the $\mathrm{K}$-shell emission region being smaller than the calculated mass-averaged plasma size (Figs. $6 \mathrm{~B}$ and $7 \mathrm{C}$ ) is consistent with this low-mass fraction contributing to the K-shell emission.

In Fig. 8B ( $-3.5 \mathrm{~ns})$, the bubble and spike structure associated with the RT instability is seen. Axial lineouts of the image show the presence of $\sim 1-\mathrm{mm}$ wavelengths that spatially merge with $\sim 3-\mathrm{mm}$ wavelengths (Fig. 8C ( $-3.5 \mathrm{~ns})$ ). These wavelengths are characteristic of those simulated by the L-RMHC (12) and E-RMHC (1), respectively. As the pinch expands after stagnation, an $\mathrm{m}=0$ sausage instability sets in (Fig. $8 \mathrm{~B}(9 \mathrm{~ns})$ ). The correspondence between the axial structure measured prior to (Fig. 8C (-3.5 ns)) and after stagnation (Fig. 8C $(9 \mathrm{~ns}))$ suggests that the RT instability seeds the sausage instability. These observations are consistent with the rz-RMHC simulations.

\section{MASS AND RADIUS VARIATION}

The variation in the widths of the total radiation pulse that was measured in the mass and radius experimental sequence is well described by E-RMHC simulations in which the magnitude of the random density perturbations $(10,11)$ is kept constant (Figs. 9A and 9E). The dashed curve in Figs. 9A, 9B, 9E, and 9F corresponds to the use of the nominal plasma emissivity in E-RMHC (15). The associated solid curve corresponds to calculations using a reduced emissivity, applied to match the measured radiation peak power at $\sim 0.6 \mathrm{mg}$ load mass (Fig. 9B), and which allows the appearance of a second peak in the radiation pulse (Fig. $6 \mathrm{~A})$. Differences between the two curves provide an estimate of the uncertainty introduced by the approximation used for the Planckian emission-absorption opacity in the E-RMHC. As shown in Figs. 9B and 9F, the measured peak power is bracketed by either calculation, which, together with the agreement in pulse 
width, provides credibility to the average hydrodynamics of the E-RMHC simulations.
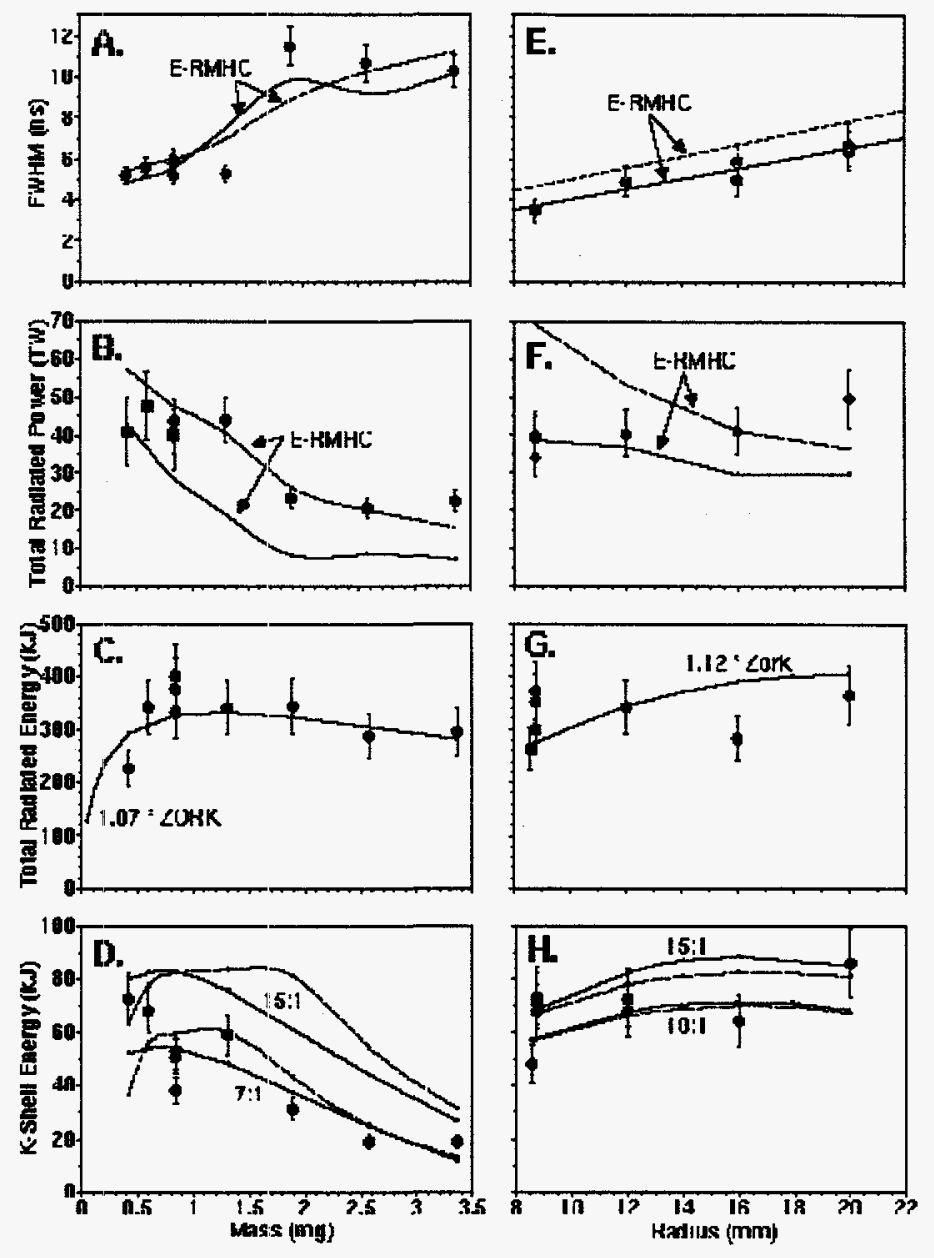

FIGURE 9. (A) Measured (uncorrected) and E-RMHC calculated (dashed curve-nominal emissivity, solid curve-reduceci emissivity) total-radiated power pulse width versus load mass. (B) Measured (uncorrected) and E-RMHC calculated (dashed curve-nominal emissivity, solid curve-reduced emissivity) total radiated power versus load mass. B corresponds to loads with less than 192 wires corrected for gap effect (Fig. 4). (C) Measured total radiated energy (uncorrected) and (1.07 \pm 0.14$)$ times the ZORK calculated kinetic energy using measured radial convergence of 20:1) versus load mass.

(D) Measured K-shell yield and that simulated by the Mosher two-level model (solid curve) and the Whitney-Giuliani scaling model (dashed curve) versus array mass for 7:1 and 15:1 radial convergences. (E) Estimated (measured using XRD filtered by $5 \mu \mathrm{m}$ Kimfol) and E-RMHC calculated (dashed curve-nominal emissivity, solid curve-reduced emissivity) total radiated power pulse width versus array radius. (F) Measured (uncorrected) and E-RMHC calculated (dashed curve-nominal emissivity, solid curvereduced emissivity) total radiated power versus array radius. (G) Measured total radiated energy (uncorrected) and $(1.12 \pm 0.28)$ times the ZORK calculated kinetic energy using measured radial convergence (which increased from 19:1 to $37: 1$ as radius increased from 8.6 to $20 \mathrm{~mm}$, respectively) versus array radius. (H) Measured K-shell yield and that 
simulated by the Mosher two-level model (solid curves) and the Whitney-Giuliani scaling model (dashed curves) versus array radius for 10:1 and 15:1 radial convergences.

These simulations show an evolution of the RT instability from short to long wavelengths similar to that seen previously (15). When a wavelength of the order of the shell thickness is reached, the bubble bursts through the shell and the shell thickness increases. As the bubbles burst, current immediately begins to flow in the low-density material between the spikes. This flow results in a continued acceleration of the shell, with only a small amount of bubble material being thrown ahead of the main body of plasma. Since the shell is still accelerating, the instability growth continues. Evolution to longer wavelengths continues again until a wavelength of the order of the new shell thickness is reached. At this time, the shell again bursts, now at longer wavelengths, which results in a significant amount of material being accelerated to the axis (Fig. 7A) and the beginning of the radiation pulse (Fig. 6A).

The total radiation yield in the simulations varies only slightly as was observed experimentally (Figs. 9C and 9G). The change in radiated power (Fig. 9B and 9F) results primarily from changes in the pulse width (Figs. 9A and 9E). In zeroth order, the pulse width is the shell thickness just prior to stagnation divided by the final average velocity. From the simulations, the lower load masses results in higher accelerations, higher final average velocities, and an increased instability growth. This growth results in a thicker shell at stagnation. At increased accelerations, however, the two-stage development of the instability produces a proportionally smaller increase in the shell thickness compared to the increase in the final average velocity. This combination results in the higher peak radiated power observed at the lower masses (Fig. 9B). Simulations of the radius variation series show that the load acceleration, the average final velocity, the instability growth, the implosion time, and the energy coupling to the load all increase as the radius increases. In the simulations, the shell thickness (due to instability growth) increased proportionately more than did the velocity, thereby lengthening the $\mathrm{x}$-ray pulse width as the array radius increased. For the simulations that used the reduced plasma emissivity, the combination of increased pulse width (Fig. 9E) and increased energy coupling to the load resulted in a radiated power that was relatively flat as the radius increases (Fig. 9F).

For the experimental data, including those of the wire number scan, the kinetic energy calculated from the ZORK model (using the measured radial convergence obtained from the FWHM of the radial lineouts of the time-integrated images of the $\mathrm{K}$-shell emission) accounts for $\sim 85 \pm 16 \%$ of the total $\mathrm{x}$-ray emission measured by the bolometer (uncorrected for spectral sensitivity and viewing angle) (Figs. 5B, 9C, and 9G). L-RMHC simulations suggest that the bolometer is sensitive to $\sim 85 \%$ of the total $\mathrm{X}$-ray emission (12). E-RMHC simulations suggest that the $\mathrm{K}$-shell lineouts overestimate the radial convergence by a factor of two (see Figs. 6B and 7C, for example) and thus the ZORK kinetic energy by $\sim 30 \%$. Therefore, about $60 \%$ of the actual total radiated energy can be accounted for by 
the OD model of kinetic energy delivered to the load. The remaining $40 \%$ is likely due to the effect of two-dimensional features including increased late-time pdV work and a small increase in the amount of Joule heating (10-12).

In Figs. 9D and 9H, the two-level (10) (solid curves) and the 1D WhitneyGiuliani (20) scaling-model (dashed curves) of K-shell yields are in qualitative agreement with those measured. These K-shell models depend only on the kinetic energy delivered to the load, the effective stagnation radius, and participating mass, and ignore the multi-dimensional dynamics of the implosion and plasma radiation after stagnation. The calculations showin in Figs. 9D and 9H used ZORK kinetic energies derived from convergence ratios varying between $7: 1$ and 15:1 with the full imploded mass. The calculated variation of yield with mass is due to the change in both temperature and density for the nearly-fixed kinetic energy (Fig. 9C). The calculated yield with radius is due primarily to the slight variation in the kinetic energy (Fig. 9G). The constancy of yield with radius reflects the optically-thick nature of the emission for aluminum and the nearly constant temperature. The need for compression ratios that are less than those calculated from lineouts may reflect unaccounted for temperature gradients (Fig. 6C) that reduce the experimental mass participation, a common problem with the one-zone scaling models. This conclusion is also reached in analysis of neon gaspuff implosions (21). This argument is supported by the L-RMHC (12) and ERMHC (Fig. 6B) simulations indicating that radial K-shell line-outs overestimate radial plasma convergence.

\section{SUMMARY}

Increasing the wire number has produced significant improvements in pinch quality, reproducibility, and x-ray output power (1). The mass variation experimental series, which was done for high-wire-number plasma-shell loads, shows that a factor of two decrease in pulse width (and an associated doubling of the radiated $\mathrm{x}$-ray power) and a greater-than-two increase in $\mathrm{K}$-shell yield occurs when the load mass transitions from masses greater than $1.9 \mathrm{mg}$ to those less than $1.3 \mathrm{mg}$, in qualitative agreement with the E-RMHC and simple $\mathrm{K}$-shell models, respectively (10). In the high-power, low-mass, plasma-shell regime, the peak power and $\mathrm{K}$-shell yields remain relatively flat with increased radius, again in agreement with the E-RMHC and the K-shell models, respectively (11). Importantly, the high quality of the implosions generated by the large-radius loads supported using such high-wire-number, plasma-shell loads for PBFA-Z $(22,23)$. The large radius enabled the initial electrical stress on PBFA-Z due to load inductance to be kept to a minimum, as the power flow in the upstream portion of the accelerator was improved. In conclusion, these experiments have conclusively demonstrated the necessity of using a large number of wires within a wire array in order to optimize the x-ray energy and power output from z-pinch implosions, and 
they represent a breakthrough in z-pinch implosion load configurations for large pulsed power generators (4).

\section{ACKNOWLEDGMENTS}

We thank T. L. Gilliland, D. O. Jobe, J. S. McGurn, P. E. Pulsifer (NRL), J. F. Seamen, K. W. Struve, W. A. Stygar, J. W. Thornhill (NRL), M. F. Vargas and the Saturn crew for their dedicated technical support; G. O. Allshouse, Y. Maron (Weizmann Institute), A. Toor (LLNL), M. Tabak (LLNL), and C. Deeney for useful discussions; M. K. Matzen, J. E. Maenchen, and D. H. McDaniel, M. E. Jones (LANL), A. Toor (LLNL), and J. Davis (NRL) for vigorous programmatic support; M. K. Matzen for carefully reviewing and L. O. Peterson for typing this manuscript. Sandia is a multiprogram laboratory operated by Sandia Corporation, a Lockheed Martin Company, for the United States Department of Energy under Contract DE-ACO4-94AL85000.

\section{REFERENCES}

1. Sanford, T. W. L., et al., Phys. Rev. Lett. 77,5063 (1996).

2. Bloomquist, D. D., et al., Proc. 6th Int. IEEE Pulsed Power Conf., Arlington, VA, ed. P. J. Turchi and B. H. Bernstein (IEEE, New York, 1987), p. 310.

3. Deeney, C., et al., "Power Enhancement by Increasing Initial Array Radius and Wire Number of Tungsten Z Pinches," submitted to Phys. Rev. E (1996).

4. Matzen, M. Keith, Phys. Plasmas 4, 1519 (1997).

5. Pereira, N. R. and Davis, J., J. Appl. Phys. 64, R1 (1988).

6. Spielman, R. B., et al., Dense z-Pinches, 3rd Intern Conf, London, UK, 1993, AIP Conf Proceed 299, editors H. Haines and A. Knight, American Institute of Physics, New York 1994, L.C. No. 93-74569, p. 404-420.

7. Deeney, C., et al., "Improved Large Diameter Wire Array Implosions from Increased Wire Array Symmetry and On-axis Mass Participation", to be submitted to Phys. Plasmas (1997).

8. Spielman, R. B., et al, 10th International Conference on High-Power Particle Beams, Prague, Czech Republic, June 10-14, 1996, ed. K. Jungwirth and J. Ullschmied, p. 150.

9. Spielman, R. B., et al., Bull. Am. Phys. Soc. 41, 1422 (1996).

10. Sanford, T. W. L., Peterson, D. L., Roderick, N. F., Mosher, D., et al., "Increased X-ray Power Generated from Low-Mass Larger-Number Aluminum-Wire-Array Z-Pinch Implosions," to be submitted to Phys. Plasmas (1997).

11. Sanford, T. W. L., Peterson, D. L., Roderick, N. F., Mosher, D., et al. "Symmetric AluminumWire Arrays Generate High-Quality z-Pinches at Large Array Diameters", to be submitted to Phys. Plasmas (1997).

12. Sanford, T. W. L., et al., Phys. Plasmas 4, 2188 (1997).

13. Sanford, T. W. L., et al., Rev. Sci. Instrum., 68, 852 (1997).

14. Hammer, J. H., et al., Phys. Plasmas 3, 2063 (1996).

15. Peterson, D. L., et al., Phys. Plasmas 3, 368 (1996).

16. Matuska, W., et al., Phys. Plasmas 3, 1415 (1996).

17. Thornhill, J. W., Whitney, K. G., and Davis, J., J. Quant. Spectrosc. Radiat. Transfer 44, 251 (1990).

18. Sanford, T. W. L., et al., Sandia National Laboratories Technical Report SAND94-0694 (June 1994).

19. Whitney, K. G., et al., "Analyzing time-resolved spectroscopic data from an azimuthally symmetric, aluminum," to be published, Phys. Rev. E (1997). 
20. Whitney, K. G., et al., J. Appl. Phys. 67, 1725 (1990).

21. D. Mosher et al., these proceedings.

22. R. B. Spielman et al., these proceedings.

23. C. Deeney et al., these proceedings. 